\title{
EDITORIAL
}

\section{The fascinating world of Soft Materials}

\author{
Published online 6 July 2020
}

Materials are everywhere: on one hand they create Earth's crust and on the other, cater to every need of the civilization. Perhaps, the importance of materials on mankind cannot be better emphasized than the fact that our pre-historic period is classified as Stone Age, Bronze Age and Iron Age, depicting materials of importance for livelihood and survival. Fast forward to the 19th Century, marked the Industrial revolution that was heralded by Steel making (Bessemer process), which eventually paved the way for the tremendous development of engineering materials.

The quest to realize materials possessing higher strength, toughness and hardness-characteristic of the traditional hard materials - while being easily processed and light weighted, led to the focus on Soft Materials. Until not so far in the past, only living matter was thought to be soft! It goes to the credit of Pierre-Gilles de Gennes, the Nobel Laureate in Physics in 1991, for bringing together a plethora of materials including colloids, gels, foams, polymers, liquid crystals, biological matter, each apparently distinct in character, under the umbrella of soft materials. The basis for unification relies on the aspect that soft materials are held together by non-covalent interactions, have forces that are typically weak and get processed at energies comparable to the thermal energy at room temperature. Being at the interface of diverse areas of chemistry, biology, chemical and materials engineering, nanotechnology and condensed matter physics, the science of soft materials is arguably the most advanced interdisciplinary fields of research. One key reason for the intense interest in soft matter systems is owing to their large and dynamic response to a range of different, and often small, stimuli leading to a wide range of smart applications and structures having shape-on-demand, exhibiting features nearly impossible to achieve with hard classical materials!

Recent years have witnessed the synthesis and applications of soft materials blossoming into a fine art and science. The resulting wide range of industrial applications has made inroads to every walk of our life, certainly making it more comfortable. Not surprisingly, a host of conventional materials like metal, ceramics and semiconductors have at least been partly replaced or preferred as soft materials, which are simultaneously stronger, lighter and cheaper. These features have been providing the impetus to discover unknown physical and chemical properties of newer materials by precisely controlling their structure and composition for tailor-made technological applications. It is a matter of pride to say that the practitioners of this field have exhibited maturity and social responsibility being conscious of the impact of their science and technology on the environment as a whole.

In this special issue, we present 27 enlightening articles written by experts in their discipline encompassing almost the entire gamut of research on soft materials, covering liquid crystals, gels, rheology, functional and self-propelled particles, orderdisorder transition, membranes, self-healing materials, transparent conducting electrodes, evaporative self-assembly, hydrophobic and slippery coatings, polymer blends and block co-polymers, thin film instability, dewetting and patterning, molecular dynamic simulation aspects and so on.

As guest editors, we are much obliged to all the contributing authors for their authoritative articles and the numerous referees for giving reviews that indubitably helped the authors to improve the quality of their manuscripts. We are beholden to Prof. G U Kulkarni, Chief Editor, for the suggestion to have a special issue on the topic, and for his constant support and guidance.

We highly appreciate the entire editorial, publishing and production teams of the Bulletin of Materials Science for their considerable efforts in bringing out this issue.

It is our belief that this issue will be found useful by the proficient in the field, besides motivating generations of students who will eventually take up research in soft materials to become the torch bearers. We do hope that any discerning reader, who does not belong to either of these groups, will also have pleasure in reading this special issue of Bulletin of Materials Science on Soft Materials.

\section{Guest editors}

\author{
KAMLENDRA AWASTHI ${ }^{1}$, RABIBRATA MUKHERJEE ${ }^{2}$ and S KRISHNA PRASAD ${ }^{3}$ (D) \\ ${ }^{1}$ Department of Physics, Malaviya National Institute of Technology, Jaipur 302017, India \\ Email: kamlendra.awasthi@gmail.com \\ ${ }^{2}$ Department of Chemical Engineering, IIT Kharagpur, Kharagpur 721302, India \\ Email: rabibrata@che.iitkgp.ac.in \\ ${ }^{3}$ Centre for Nano and Soft Matter Sciences, Bengaluru 560 013, India \\ Email: skpras@gmail.com
}

